\title{
On the determination of oxygen abundances in chromospherically active stars ${ }^{\star}, \star \star$
}

\author{
T. Morel and G. Micela \\ Istituto Nazionale di Astrofisica, Osservatorio Astronomico di Palermo G. S. Vaiana, Piazza del Parlamento 1, 90134 Palermo, \\ Italy \\ e-mail: morel@astropa.unipa.it \\ Received 2 March 2004 / Accepted 29 April 2004

\begin{abstract}
We discuss oxygen abundances derived from [ $\left.\mathrm{O}_{\mathrm{I}}\right] \lambda 6300$ and the $\mathrm{O}_{\mathrm{I}}$ triplet in stars spanning a wide range in chromospheric activity level, and show that these two indicators yield increasingly discrepant results with higher chromospheric/coronal activity measures. While the forbidden and permitted lines give fairly consistent results for solar-type disk dwarfs, spuriously high O I triplet abundances are observed in young Hyades and Pleiades stars, as well as in individual components of RS CVn binaries (up to $1.8 \mathrm{dex}$ ). The distinct behaviour of the [O I]-based abundances which consistently remain near-solar suggests that this phenomenon mostly results from large departures from LTE affecting the O I triplet at high activity level that are currently unaccounted for, but also possibly from a failure to adequately model the atmospheres of K-type stars. These results suggest that some caution should be exercised when interpreting oxygen abundances in active binaries or young open cluster stars.
\end{abstract}

Key words. stars: abundances - stars: activity - line: formation

\section{Introduction}

The determination of oxygen abundances in distinct stellar populations (bulge, thin/thick disk and halo) is of fundamental importance for a better understanding of the chemical evolution of the Galaxy and its formation history (e.g., McWilliam 1997). On the other hand, oxygen is a key ingredient in evolution codes, as it affects the energy generation and opacity in stellar interiors. This is of particular relevance for issues related to the lithium depletion in open cluster stars, for instance (e.g., Piau \& Turck-Chièze 2002).

For practical reasons, oxygen abundances have been traditionally determined from transitions in the optical domain, either the $7774 \AA$-O I triplet or the forbidden [O I] $\lambda 6300$ and [O I] $\lambda 6363$ lines (these spectral diagnostics can be complemented by molecular UV and IR OH bands: e.g., Boesgaard et al. 1999). Unfortunately, the former is bound to be affected by significant, yet poorly-constrained non-LTE (NLTE) effects. Although the forbidden lines are insensitive to departures from LTE, they are generally very weak and become virtually immeasurable in metal-poor dwarfs, which are potentially among the most interesting targets. Additionally, they also suffer from blending with weak $\mathrm{CN}$ or $\mathrm{Ni}$ lines (see below). Both indicators are very sensitive to the effective temperature and, to a lesser extent, to the surface gravity assumed. Furthermore, the

\footnotetext{
* Based on observations collected at the European Southern Observatory, Chile (Proposals 64.L-0249 and 071.D-0260).

$\star \star$ Table 2 is only available in electronic form at http://www.edpsciences.org
}

treatment of granulation may also be important (Asplund et al. 2004). The long-standing discrepancy often observed between these two indicators (the permitted lines generally yielding systematically higher abundances than the forbidden transitions) likely stems from these caveats, although the cause of this disparity is still, despite much work, not well understood (e.g., Fulbright \& Johnson 2003).

During the course of our abundance study of a sample of single-lined RS CVn binaries (Morel et al. 2003, hereafter M03; Morel et al. 2004, hereafter M04), we noticed a dramatic discrepancy between the oxygen abundances given by [O I] $\lambda 6300$ and the O I triplet, with the latter yielding suspiciously high values. Using these observations, supplemented by data for Pleiades, Hyades and field FG dwarfs, here we show that the $[\mathrm{O} \mathrm{I}]-$ and $\mathrm{O} \mathrm{I}-$ based abundances diverge with increasing stellar activity level.

\section{Observational data}

Spectra of 14 single-lined chromospherically active binaries from the list of Strassmeier et al. (1993) were obtained in 2000 and 2003 at the ESO 1.5/2.2-m telescopes at La Silla (Chile) with the echelle spectrograph FEROS (resolving power $R$ $48000)$. The spectral range covered is $3600-9200 \AA$, hence enabling a simultaneous coverage of $\mathrm{Ca}$ II $\mathrm{H}+\mathrm{K}$, [O I] $\lambda 6300$ and the $\mathrm{O}_{\mathrm{I}}$ triplet (the extremely weak [O I] $\lambda 6363$ line could not be reliably measured in our spectra). We also observed 
a control sample made up of 7 stars with similar characteristics (i.e. K2-G8 subgiants), but with low X-ray luminosities (Hünsch et al. 1998a).

We refer the reader to M03 and M04 for details on the reduction procedure and abundance analysis. Briefly, the effective temperature and surface gravity were derived from the excitation and ionization equilibrium of the iron lines, while the microturbulent velocity was determined by requiring the $\mathrm{Fe}_{\mathrm{I}}$ abundances to be independent of the line strength. The oxygen abundances were derived from a differential analysis using plane-parallel, line-blanketed LTE Kurucz atmospheric models (with a length of the convective cell over the pressure scale height, $\alpha=l / H_{\mathrm{p}}=0.5$, and no overshoot) and the current version of the MOOG software. The choice of another 1-D model (e.g. MARCS) is very unlikely to substantially affect the conclusions presented in the following (see Fulbright \& Johnson 2003). All $g f$-values were calibrated with a high $\mathrm{S} / \mathrm{N}$ moonlight spectrum acquired with the same instrumental setup. The values adopted for the oxygen features and the measured equivalent widths (EWs) are given in Table 1 . The $E W \mathrm{~s}$ of [O I] $\lambda 6300$ have been corrected for the contribution of the high-excitation Ni I $\lambda 6300.339$ line $(\chi=4.27 \mathrm{eV})$. The corresponding $E W$ was estimated by a curve-of-growth analysis using the derived atmospheric parameters and $\mathrm{Ni}$ abundances (M03, M04), and assuming $\log g f=-2.31$ (Allende Prieto et al. 2001). To be consistent with data for Pleiades stars (Schuler et al. 2004), we use this value instead of a recent (and presumably more accurate) laboratory determination $(\log g f=$ -2.11; Johansson et al. 2003). Albeit extremely weak (typically $E W \sim 3-4 \mathrm{~mA}$ ), the Ni line may contribute up to $15 \%$ to the total $E W$. A similar procedure was used to estimate the oscillator strength of [O I $] 26300$ from the solar spectrum.

The bulk of the oxygen abundances for Pleiades stars come from Schuler et al. (2004), with the exception of HII 676 (King et al. 2000). For Hyades stars, we use the data of García López et al. (1993) and King \& Hiltgen (1996). For the nearby main sequence FG stars, we restrict ourselves to objects with measurements in both [O I] $\lambda 6300$ and the $\mathrm{O}_{\mathrm{I}}$ triplet (Bensby et al. 2004; King \& Boesgaard 1995; Reddy et al. 2003). Among these sources, only King \& Boesgaard (1995) and King \& Hiltgren (1996) did not take into account the Ni I $\lambda 6300.3$ line. Their [OI] $\lambda 6300$ abundances are thus likely to be slightly overestimated. To avoid oxygen overabundances arising from contamination from Type II supernova ejecta, care has been taken to only select stars with solar metallicities and/or kinematical properties typical of the thin disk population. The oxygen abundances from the literature have been rescaled to our adopted oxygen and iron solar abundances. To be consistent with Kurucz models and opacities, we assumed $\log \epsilon_{\odot}(\mathrm{O})=$ 8.93 and $\log \epsilon_{\odot}(\mathrm{Fe})=7.67$. In addition, all abundances given by the $\mathrm{O}_{\text {I }}$ triplet have been uniformly converted into NLTE values using the corrections of Gratton et al. (1999). The abundances are systematically revised downwards by a small amount never exceeding 0.13 dex.

The activity index, $R_{\mathrm{HK}}^{\prime}$, defined as the radiative loss in the $\mathrm{Ca}$ II $\mathrm{H}+\mathrm{K}$ lines in units of the bolometric luminosity (after correction for photospheric contribution), was used as a primary indicator of chromospheric activity. This quantity was derived
Table 1. Oscillator strengths, first excitation potentials and $E W$ s of the oxygen features (in $\mathrm{m} \AA$ ). A blank indicates that the $E W$ was not reliably measurable because of an unsatisfactory Gaussian fit or cosmic rays/telluric features affecting the line profile. The latter issue is particularly relevant in the case of [O I ] $\lambda 6300$ (we used the telluric atlas of Hinkle et al. 2000).

\begin{tabular}{lcccc}
\hline \hline$\lambda(\AA)$ & $6300.304^{a}$ & 7771.944 & 7774.166 & 7775.388 \\
\hline $\log g f$ & -9.778 & 0.297 & 0.114 & -0.064 \\
$\chi(\mathrm{eV})$ & 0.000 & 9.147 & 9.147 & 9.147 \\
\hline HD 28 & & 35.8 & 36.5 & 32.0 \\
HD 1227 & & 58.1 & 52.7 & \\
HD 4482 & 25.1 & 68.2 & & 47.3 \\
HD 10909 & 18.7 & 65.3 & 54.4 & 38.7 \\
HD 17006 & & 64.4 & & \\
HD 19754 & 21.8 & & 62.5 & 55.7 \\
HD 72688 & & 81.6 & & 58.3 \\
HD 83442 & & 84.0 & 75.8 & \\
HD 113816 & 20.8 & 92.3 & & \\
HD 118238 & & 125.1 & 120.7 & \\
HD 119285 & & 93.8 & 84.2 & 53.9 \\
HD 154619 & 19.2 & 62.9 & 59.2 & 45.6 \\
HD 156266 & 34.3 & & & \\
HD 181809 & 13.4 & 64.5 & & 44.8 \\
HD 182776 & & & 95.7 & \\
HD 202134 & 22.7 & 86.3 & & \\
HD 204128 & & 82.1 & & \\
HD 205249 & & 95.4 & 92.4 & 64.1 \\
HD 211391 & 27.7 & 60.0 & & \\
HD 217188 & & 76.8 & & \\
HD 218527 & & 52.2 & 49.8 & \\
\hline
\end{tabular}

${ }^{a}$ Corrected for the contribution of $\mathrm{Ni}$ I $\lambda 6300.339$ (see text).

from our spectra following Linsky et al. (1979). In view of the $(V-R)$ and $(V-I)$ colour anomalies exhibited by active stars (see M04), we did not use the observed $(V-R)$ value in the derivation of the absolute flux in the 3925-3975 $\AA$ wavelength range, $\mathcal{F}_{50}$. Instead, we computed the colour appropriate for a star with the derived effective temperature and iron abundance (Eq. (5) of Alonso et al. 1999). The bolometric luminosities were estimated from theoretical isochrones. The $R_{\mathrm{HK}}^{\prime}$ data for disk dwarfs were gathered from the literature (Soderblom 1985; Henry et al. 1996; Wright et al. 2004). The data for Hyades stars come from Paulson et al. (2002). For 8 stars in this sample, there is a good agreement with previous measurements (Duncan et al. 1984). We also define $R_{\mathrm{X}}$, which is given as the ratio between the X-ray (in the ROSAT [0.1-2.4 keV] energy band) and bolometric luminosities. More details regarding the derivation of $R_{\mathrm{HK}}^{\prime}$ and $R_{\mathrm{X}}$ can be found in M03. The oxygen abundances and activity indices, as well as the source of the data, are given in Table 2 (only available in electronic form).

\section{Results}

Figure 1 shows the oxygen abundances yielded by [O I] $\lambda 6300$ and the $\mathrm{O}_{\mathrm{I}}$ triplet, as a function of the activity indices $R_{\mathrm{HK}}^{\prime}$ 


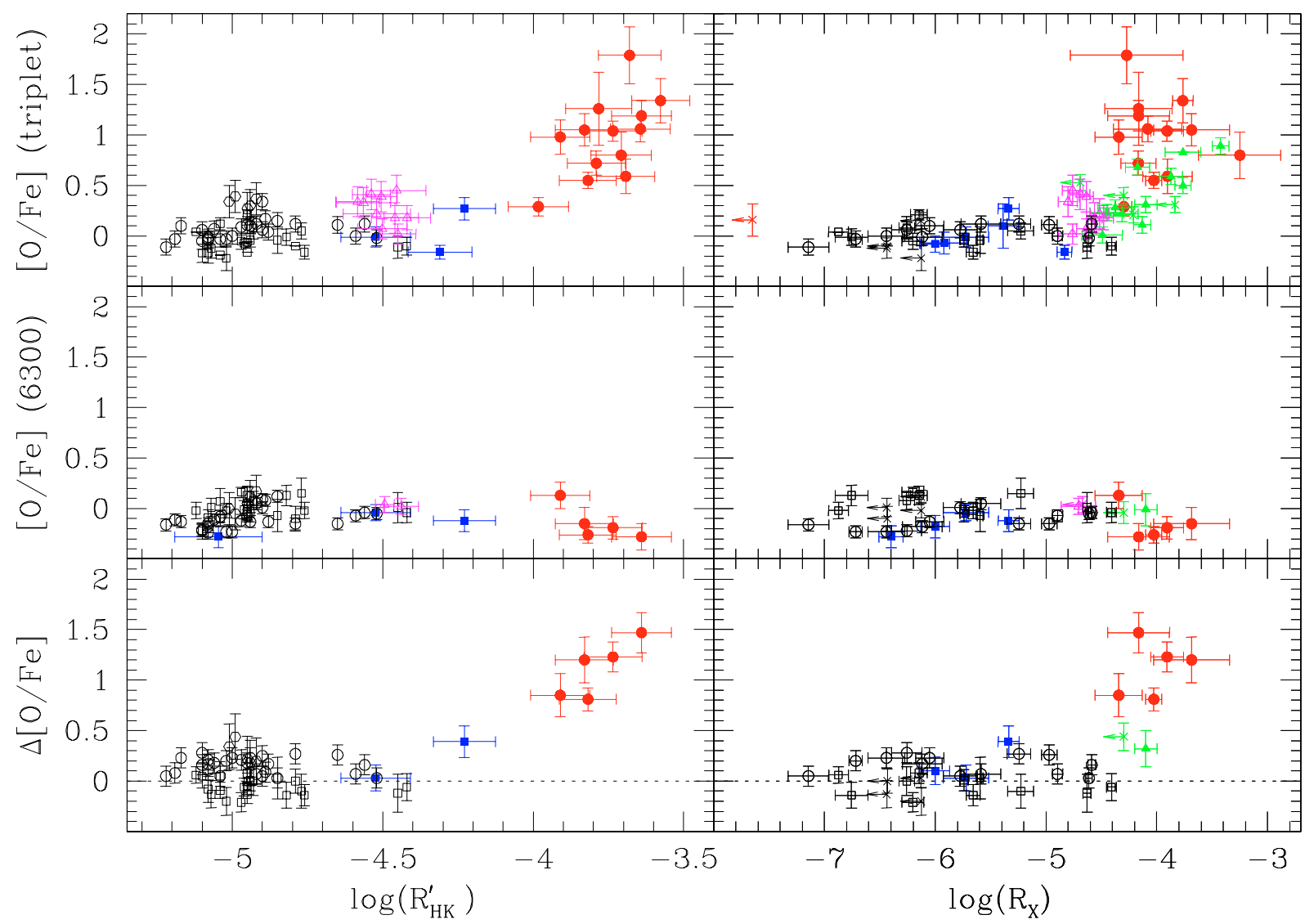

Fig. 1. Oxygen abundances as a function of the activity indices $R_{\mathrm{HK}}^{\prime}$ and $R_{\mathrm{X}}$. The bottom panels show the difference between [O/Fe] given by the $\mathrm{O}_{\mathrm{I}}$ triplet and the [O I] $\lambda 6300$ line. Filled circles: RS CVn binaries (M03; M04), filled squares: field subgiants (M04), filled triangles: Pleiades stars (King et al. 2000; Schuler et al. 2004), open triangles: Hyades stars (García López et al. 1993; King \& Hiltgen 1996), open circles, squares and hexagons: disk dwarfs (from Bensby et al. 2004; King \& Boesgaard 1995; Reddy et al. 2003, respectively). The colour coding in the online version of this journal is the following: red: RS CVn binaries, blue: field subgiants, green: Pleiades stars, magenta: Hyades stars. The crosses in the right-hand panels are upper limits.

and $R_{\mathrm{X}}$. As can be seen, the abundances given by both set of lines are in fair agreement for solar-type field stars. However, there is a steeply increasing discrepancy as one goes to higher activity levels. In sharp contrast to the behaviour of the $\mathrm{O}_{\mathrm{I}}$ triplet, the $\left[\mathrm{O}_{\mathrm{I}}\right]$ 16300-based abundances remain roughly solar even for the most active stars. There is a hint that the Pleiades stars exhibit lower triplet abundances than the active binaries at a given $R_{\mathrm{X}}$ value. Several inconsistencies (e.g. temperature scale) are likely to introduce systematics when comparing abundances taken from various works in the literature. However, such an offset might arise from a temperature effect (see Sect. 4). We note that the choice of the atomic data is of little relevance here. The oscillator strengths adopted in the various literature sources (including our values) lie within 0.08 dex, i.e. a value translating into abundance differences typically comparable to the uncertainties.

We cannot rule out the existence of a systematic underestimation of the iron abundances (at the $\sim 0.1-0.2$ dex level) in the RS CVn binaries (M04). Although this would evidently significantly affect the $[\mathrm{O} / \mathrm{Fe}]$ values, the difference between the abundances derived from the permitted and forbidden lines is independent of the iron content and is thus a robust indicator of the influence of chromospheric activity.
The correlation between the O I-based abundances and $R_{\mathrm{X}}$ is remarkably tight considering the following points ${ }^{1}$. Firstly, we recall that the measurements of the oxygen abundances and $\mathrm{X}$-ray luminosities are not, contrary to the $\mathrm{Ca}$ II $\mathrm{H}+\mathrm{K}$ data, cotemporal. In addition to flare-like events, the X-ray emission in RS CVn binaries and young open cluster stars can be intrinsically variable on long timescales (e.g., Kashyap \& Drake 1999; Micela et al. 1996). Secondly, in contrast to the X-ray data which diagnose the coronal regions, the $\mathrm{Ca}$ II $\mathrm{H}+\mathrm{K}$ lines are spectral diagnostics of the lower chromosphere and are as such better probes of the physical conditions prevailing in vicinity of the stellar photosphere.

\section{Discussion}

As mentioned previously, the [O I] and $\mathrm{O}_{\mathrm{I}}$ lines are very sensitive to the choice of the atmospheric model. It is therefore conceivable that the trend observed in Fig. 1 is actually an artefact of systematics in the determination of the atmospheric

\footnotetext{
1 In the Pleiades dataset alone, [O/Fe] (triplet) is correlated with $R_{\mathrm{X}}$ at a confidence level exceeding $98 \%$, whether or not upper limits are considered (we made us of the Kendall's $\tau$ method; Isobe et al. 1986).
} 
parameters. In the case of the active binaries, our excitation temperatures appear marginally higher than values derived from $T_{\text {eff- }}(B-V)$ calibrations based on the infrared flux method (Alonso et al. 1999): $\left\langle T_{\text {exc }}-T_{\text {colour }}\right\rangle=+80 \pm$ $46 \mathrm{~K}$ (M04). On the other hand, our surface gravities derived from the ionization equilibrium of the iron lines tend to be lower than values derived from theoretical isochrones $\left(\left\langle\log g_{\text {ioni }}-\log g_{\text {iso }}\right\rangle=-0.21 \pm 0.06 \mathrm{dex}\right)$. Such zero-point offsets may have various causes (e.g. NLTE effects, identification of model label temperatures with values derived from integrated flux). We examined the potential impact of systematic errors of this magnitude on the resulting oxygen abundances, as well as the effect of the treatment of convective energy transport (overshoot option switched on/off), as it modifies the $T(\tau)$ relation in the deepest photospheric layers where the $\mathrm{O}_{\mathrm{I}}$ triplet is formed. Tests were also performed with models characterized by different opacities (by means of an enrichment in $\alpha$ elements). Since the excitation and ionization equilibrium of the iron lines must be simultaneously satisfied, note that changes in $T_{\text {eff }}$ are necessarily accompanied by variations in $\log g$ and vice versa. As expected, errors in $T_{\text {eff }}$ and $\log g$ significantly bias the $[\mathrm{O} / \mathrm{Fe}]$ determinations (see Table 3). However, resolving the discrepancy between the oxygen indicators would require alterations of these quantities well beyond any reasonable uncertainty. More importantly, the differences between the excitation/ $(B-V)$-colour temperatures and the ionization/isochrone gravities are not correlated with the activity indices. This rules out the possibility that the activity trend results from an increasingly underestimated temperature at high activity levels, for instance. The presence of a faint, cooler companion is also not thought to significantly affect our $T_{\text {eff determinations (Katz et al. 2003). }}$.

Let us now examine the potential importance of physical processes causally related to activity. Following Neff et al. (1995), we investigated the effect of photospheric spots by carrying out an abundance analysis on a composite, synthetic Kurucz spectrum of a fictitious star with $T_{\text {eff }}=4830 \mathrm{~K} \mathrm{cov-}$ ered by cooler regions $\left(T_{\text {eff }}=3830 \mathrm{~K}\right)$ with different covering factors, $f_{s}$ (see M03). Although the effect is appreciable, we find that the oxygen discrepancy only increases by 0.08 and 0.12 dex for $f_{s}=30$ and $50 \%$, respectively. We assessed in M03 the importance of chromospheric heating in inducing abundance anomalies by altering the temperature structure of the Kurucz models: a temperature gradient reversal in the outermost regions was incorporated, as well as a heating (up to $190 \mathrm{~K}$ ) of the deeper photospheric layers (see Fig. 3 of M03). We considered two model chromospheres meant to be representative of K-type giants (Kelch et al. 1978) and RS CVn binaries (Lanzafame et al. 2000). A LTE abundance analysis was then performed on HD 10909 using a classical Kurucz model and these two sets of model atmospheres. To fulfil the excitation and ionization equilibrium of the iron lines, it was necessary in the two latter cases to use an "underlying" Kurucz model with a much lower effective temperature and gravity. Here we adopt a different approach by keeping the parameters of the atmospheric model unchanged (only the microturbulence was adjusted). Because of their inverse sensitivity to temperature changes, it comes as no surprise that
Table 3. Effect of changes in the stellar parameters of HD 202134 on the oxygen abundances. $\Delta[\mathrm{O} / \mathrm{Fe}]$ is defined as $[\mathrm{O} / \mathrm{Fe}]$ (triplet)-[O/Fe] (6300).

\begin{tabular}{lcccc}
\hline \hline & {$[\mathrm{Fe} / \mathrm{H}]$} & $\begin{array}{c}{[\mathrm{O} / \mathrm{Fe}]} \\
6300\end{array}$ & $\begin{array}{c}{[\mathrm{O} / \mathrm{Fe}]} \\
\text { triplet }\end{array}$ & $\Delta[\mathrm{O} / \mathrm{Fe}]$ \\
\hline$\Delta T_{\text {eff }}=+150 \mathrm{~K}$ & +0.08 & +0.14 & -0.18 & -0.32 \\
$\Delta \log g=+0.25 \mathrm{dex}[\mathrm{cgs}]$ & +0.04 & +0.08 & -0.10 & -0.18 \\
$\Delta \xi=+0.20 \mathrm{~km} \mathrm{~s}^{-1}$ & -0.08 & +0.08 & +0.01 & -0.07 \\
$\Delta[\alpha / \mathrm{Fe}]=-0.2$ & -0.01 & -0.01 & +0.01 & +0.02 \\
with overshooting & +0.00 & +0.01 & +0.01 & +0.00 \\
\hline
\end{tabular}

merging a chromospheric component on the Kurucz model leads to a better agreement between the oxygen indicators. However, the discrepancy is "only" reduced by 0.17 and 0.28 dex for the model chromospheres of Kelch et al. (1978) and Lanzafame et al. (2000), respectively. This seems insufficient to account for the observations.

Apart from a global temperature rise, however, it is likely that a chromosphere would primarily manifest itself by overionization/overexcitation effects. In the case of the Sun, for instance, the solar chromosphere must be taken into account when studying the NLTE line formation of the OI triplet and, in particular, to reproduce the centre-to-limb behaviour of these features (Takeda 1995). To our knowledge, all comprehensive NLTE line formation studies published to date are based on conventional atmospheric models without a chromospheric component. Several investigations have concentrated on FG dwarfs, but considerably less attention has been unfortunately paid to cooler stars. The NLTE corrections of Gratton et al. (1999) can be very large for warm, low gravity stars (up to $1 \mathrm{dex}$ ), but are modest for K-type subgiants (less than 0.13 dex for the stars in our sample; similar results were obtained by Takeda 2003). When applied to our abundances, they only slightly reduce the disparity between the oxygen indicators. Yong et al. (2004) recently demonstrated the existence of dramatic overionization effects in Pleiades dwarfs, with an increasing discrepancy between the Fe I- and Fe II-based abundances with decreasing $T_{\text {eff }}$. This highlights the unexpectedly large magnitude of the NLTE effects in cool, active stars. Since the O I triplet is known to be liable to large departures from LTE, it is tempting to associate the oxygen abundance anomalies observed in Pleiades stars to this phenomenon (Schuler et al. 2004). The fact that [O I] $\lambda 6300$, which does not experience these effects, consistently yields roughly near-solar abundances in the active binaries supports a similar interpretation in our sample (see Fig. 1).

Tomkin et al. (1992) reported an increasing discrepancy between the oxygen indicators with decreasing effective temperatures in halo main sequence FG stars. In a similar vein, Schuler et al. (2004) found that cool stars in the Pleiades exhibit higher O I-based oxygen abundances. This may be primarily an activity effect in view of the tight correlation between $(B-V)_{0}$ and $R_{\mathrm{X}}$ (Micela et al. 1999). There is also some evidence in our data for a relationship between $T_{\text {eff }}$ and [O/Fe] (Fig. 2). This might arise from the fact that the most active stars (i.e. 


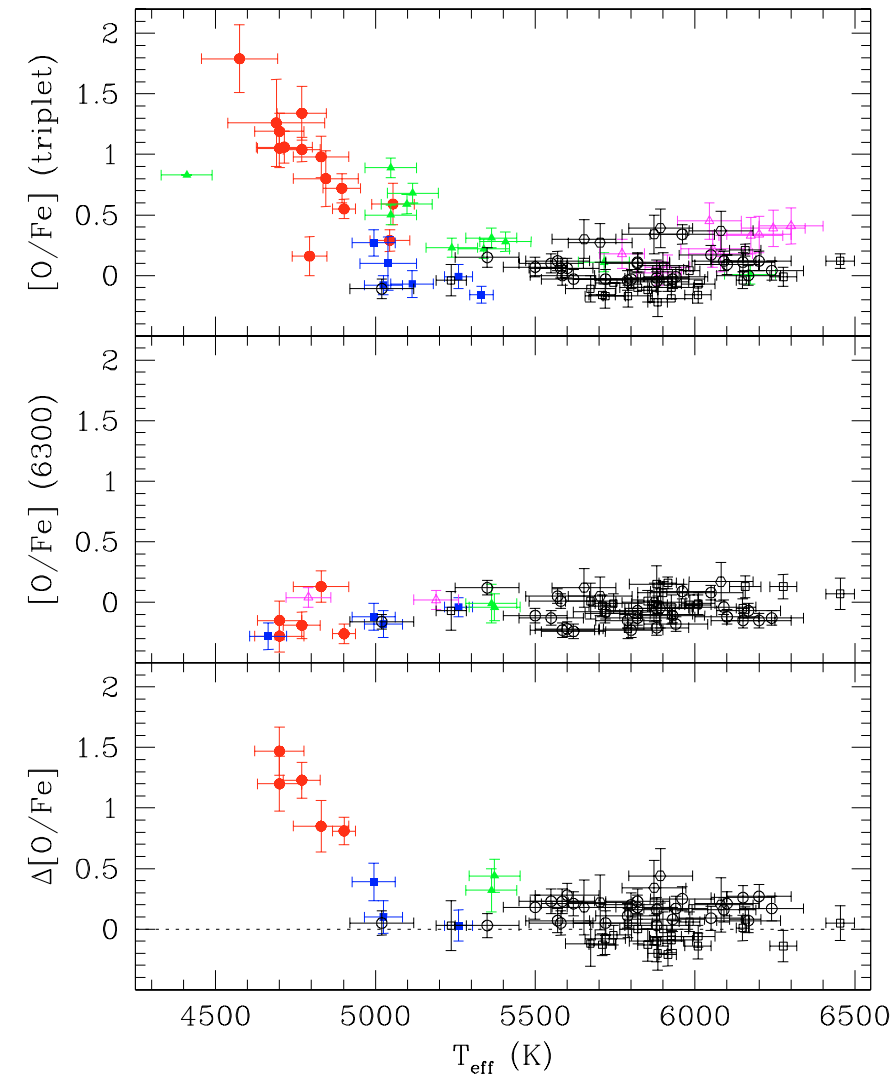

Fig. 2. Oxygen abundances as a function of the effective temperature. The bottom panel shows the difference between $[\mathrm{O} / \mathrm{Fe}]$ given by the $\mathrm{O}_{\mathrm{I}}$ triplet and the [O I] $\lambda 6300$ line. Symbols as in Fig. 1.

the RS CVn binaries) turn out to be the coolest objects in our sample. It is worth noting that a similar relation also seems to hold for presumably inactive, metal-poor (sub)giants (see Fig. 10 of Fulbright \& Johnson 2003), although the difference is much more marked here $(\Delta[\mathrm{O} / \mathrm{Fe}]=1.5$ against 0.5 dex at $\left.T_{\text {eff }} \sim 4700 \mathrm{~K}\right)$. If confirmed at near-solar metallicity, this trend would suggest that the same mechanism acts in both samples of active and inactive cool stars to yield spuriously high abundances from the $\mathrm{O}$ i triplet.

To summarize, we cannot rule out either of these two (non mutually exclusive) possibilities: (a) the discrepancy between the oxygen indicators results from inadequacies in the atmospheric models for cool stars (e.g. inappropriate temperature structure in the deepest layers; Carretta et al. 2000; Israelian et al. 2004), this problem becoming more acute for chromospherically active stars; or (b) NLTE effects are dramatically underestimated in cool, active stars and, to a lesser extent, in their inactive analogues.

\section{Summary}

As discussed in this paper, [O I] $\lambda 6300$ appears relatively free of the problems affecting the $\mathrm{O}$ I triplet. This makes it a much better oxygen abundance indicator in stars with a very high level of chromospheric activity, such as members of young open clusters or active, tidally-locked binaries. Although using molecular $\mathrm{OH}$ bands may constitute an alternative, having to rely solely on the [OI] $\lambda 6300$ line appears rather unfortunate considering the weakness of this feature in open cluster dwarfs, for instance $(E W \lesssim 10 \mathrm{~m} \AA$ ). On a more optimistic note, our observations have unveiled a missing ingredient in our modelling of the $\mathrm{O}_{\mathrm{I}}$ triplet in active, cool stars. Although challenging, more progress on this issue can be expected from theoretical studies: more realistic models for K-type stars (e.g. incorporating thermal inhomogeneities; Nissen et al. 2002) and detailed NLTE calculations applied to chromospherically active stars.

Acknowledgements. This research was supported through a European Community Marie Curie Fellowship (No. HPMD-CT-2000-00013). G.M. acknowledges financial support from MIUR (Ministero della Istruzione, dell'Università e della Ricerca). We wish to thank Dr. S. C. Schuler for making his manuscript available to us prior to publication and the anonymous referee for useful comments. This research made use of NASA's Astrophysics Data System Bibliographic Services and the Simbad database operated at CDS, Strasbourg, France.

\section{References}

Allende Prieto, C., Lambert, D. L., \& Asplund, M. 2001, ApJ, 556, L63

Alonso, A., Arribas, S., \& Martínez-Roger, C. 1999, A\&AS, 140, 261

Asplund, M., Grevesse, N., Sauval, A. J., Allende Prieto, C., \& Kiselman, D. 2004, A\&A, 417, 751

Bensby, T., Feltzing, S., \& Lundström, I. 2004, A\&A, 415, 155

Boesgaard, A. M., King, J. R., Deliyannis, C. P., \& Vogt, S. S. 1999, AJ, 117, 492

Carretta, E., Gratton, R. G., \& Sneden, C. 2000, A\&A, 356, 238

Cayrel, R., Cayrel de Strobel, G., \& Campbell, B. 1985, A\&A, 146, 249

Crawford, D. L., \& Perry, C. L. 1976, AJ, 81, 419

Dempsey, R. C., Linsky, J. L., Fleming, T. A., \& Schmitt, J. H. M. M. 1993, ApJS, 86, 599

Dempsey, R. C., Linsky, J. L., Fleming, T. A., \& Schmitt, J. H. M. M. 1997, ApJ, 478, 358

Duncan, D. K., Baliunas, S. L., Noyes, R. W., et al. 1984, PASP, 96, 707

Flower, P. J. 1996, ApJ, 469, 355

Fulbright, J. P., \& Johnson, J. A. 2003, ApJ, 595, 1154

García López, R. J., Rebolo, R., Herrero, A., \& Beckman, J. E. 1993 , ApJ, 412, 173

Gratton, R. G., Carretta, E., Eriksson, K., \& Gustafsson, B. 1999, A\&A, 350, 955

Henry, T. J., Soderblom, D. R., Donahue, R. A., \& Baliunas, S. L. 1996, AJ, 111, 439

Hertzsprung, E. 1947, Ann. Sternw. Leiden, 19, Part 1

Hinkle, K., Wallace, L., Valenti, J., \& Harmer, D. 2000, Visible and Near Infrared Atlas of the Arcturus Spectrum 3727-9300 ̊ (San Francisco: ASP)

Hünsch, M., Schmitt, J. H. M. M., \& Voges, W. 1998a, A\&AS, 127, 251

Hünsch, M., Schmitt, J. H. M. M., \& Voges, W. 1998b, A\&AS, 132, 155

Hünsch, M., Schmitt, J. H. M. M., Sterzik, M. F., \& Voges, W. 1999, A\&AS, 135, 319

Isobe, T., Feigelson, E. D., \& Nelson, P. I. 1986, ApJ, 306, 490

Israelian, G., Shchukina, N., Rebolo, R., et al. 2004, A\&A, 419, 1095

Johansson, S., Litzén, U., Lundberg, H., \& Zhang, Z. 2003, ApJ, 584, L107 
Kashyap, V., \& Drake, J. J. 1999, ApJ, 524, 988

Katz, D., Favata, F., Aigrain, S., \& Micela, G. 2003, A\&A, 397, 747

Kelch, W. L., Linsky, J. L., Basri, G. S., et al. 1978, ApJ, 220, 962

King, J. R., \& Boesgaard, A. M. 1995, AJ, 109, 383

King, J. R., \& Hiltgen, D. D. 1996, AJ, 112, 2650

King, J. R., Soderblom, D. R., Fischer, D., \& Jones, B. F. 2000, ApJ, 533,944

Lanzafame, A. C., Busà, I., \& Rodonò, M. 2000, A\&A, 362, 683

Linsky, J. L., Worden, S. P., McClintock, W., \& Robertson, R. M. 1979, ApJS, 41, 47

McWilliam, A. 1997, ARA\&A, 35, 503

Micela, G., Sciortino, S., Kashyap, V., Harnden, Jr., F. R., \& Rosner, R. 1996, ApJS, 102, 75

Micela, G., Sciortino, S., Harnden, Jr., F. R., et al. 1999, A\&A, 341, 751

Morel, T., Micela, G., Favata, F., Katz, D., \& Pillitteri, I. 2003, A\&A, 412, 495 (M03)

Morel, T., Micela, G., Favata, F., \& Katz, D. 2004, A\&A, in press [arXiv: astro-ph/0406348] (M04)

Neff, J. E., O’Neal, D., \& Saar, S. H. 1995, ApJ, 452, 879

Neuhäuser, R., Walter, F. M., Covino, E., et al. 2000, A\&AS, 146, 323

Nissen, P. E. 1988, A\&A, 199, 146

Nissen, P. E., Primas, F., Asplund, M., \& Lambert, D. L. 2002, A\&A, 390,235

Paulson, D. B., Saar, S. H., Cochran, W. D., \& Hatzes, A. P. 2002, AJ, 124,572
Piau, L., \& Turck-Chièze, S. 2002, ApJ, 566, 419

Reddy, B. E., Tomkin, J., Lambert, D. L., \& Allende Prieto, C. 2003, MNRAS, 340, 304

Schmitt, J. H. M. M. 1997, A\&A, 318, 215

Schmitt, J. H. M. M., \& Liefke, C. 2004, A\&A, 417, 651

Schuler, S. C., King, J. R., Hobbs, L. M., \& Pinsonneault, M. H. 2004, ApJ, 602, L117

Schwan, H. 1991, A\&A, 243, 386

Schwope, A. D., Hasinger, G., Lehmann, I., et al. 2000, Astron. Nachr., 321, 1

Soderblom, D. R. 1985, AJ, 90, 2103

Stauffer, J. R., Caillault, J.-P., Gagné, M., Prosser, C. F., \& Hartmann, L. W. 1994, ApJS, 91, 625

Stern, R. A., Schmitt, J. H. M. M., \& Kahabka, P. T. 1995, ApJ, 448, 683

Strassmeier, K. G., Hall, D. S., Fekel, F. C., \& Scheck, M. 1993, A\&AS, 100, 173

Takeda, Y. 1995, PASJ, 47, 463

Takeda, Y. 2003, A\&A, 402, 343

Tomkin, J., Lemke, M., Lambert, D. L., \& Sneden, C. 1992, AJ, 104, 1568

van Bueren, H. G. 1952, Bull. Astron. Inst. Netherlands, 11, 385

Wright, J. T., Marcy, G. W., Butler, R. P., \& Vogt, S. S. 2004, ApJS, 152,261

Yong, D., Lambert, D. L., Allende Prieto, C., \& Paulson, D. B. 2004, ApJ, 603, 697 
T. Morel and G. Micela: Oxygen abundances in chromospherically active stars, Online Material p 1

\section{Online Material}


T. Morel and G. Micela: Oxygen abundances in chromospherically active stars, Online Material p 2

Table 2. Oxygen abundances and activity indices. The $[\mathrm{O} / \mathrm{Fe}]$ data from the literature were rescaled to our adopted iron and oxygen solar abundances $\left(\log \epsilon_{\odot}[\mathrm{Fe}]=7.67\right.$ and $\left.\log \epsilon_{\odot}[\mathrm{O}]=8.93\right)$, and corrected for departures from LTE in the case of the O I triplet (Gratton et al. 1999). We use the values of Schuler et al. (2004) derived without convective overshoot ("NOVER").

\begin{tabular}{|c|c|c|c|c|c|c|c|c|c|c|}
\hline $\mathrm{ID}^{a}$ & $\begin{array}{l}T_{\text {eff }} \\
(\mathrm{K})\end{array}$ & {$[\mathrm{Fe} / \mathrm{H}]^{b}$} & {$[\mathrm{O} / \mathrm{Fe}](6300)$} & {$[\mathrm{O} / \mathrm{Fe}]$ (Triplet) } & Ref. & $\log \left(R_{\mathrm{HK}}^{\prime}\right)^{c}$ & Ref. & $\begin{array}{c}\log \left(L_{\mathrm{X}}\right)^{d} \\
\left(\mathrm{erg} \mathrm{s}^{-1}\right)\end{array}$ & $\log \left(R_{\mathrm{X}}\right)^{d}$ & Ref. \\
\hline \multicolumn{11}{|c|}{ RS CVn binaries } \\
\hline HD 28 & 4794 & $0.01 \pm 0.08$ & & $0.16 \pm 0.16$ & 1 & & & $<27.34$ & $<-7.65$ & 14 \\
\hline HD 10909 & 4830 & $-0.41 \pm 0.09$ & $0.13 \pm 0.13$ & $0.98 \pm 0.17$ & 2 & $-3.91 \pm 0.10$ & 2 & $30.26 \pm 0.21$ & $-4.34 \pm 0.21$ & 15 \\
\hline HD 19754 & 4700 & $-0.39 \pm 0.08$ & $-0.15 \pm 0.16$ & $1.05 \pm 0.16$ & 1 & $-3.83 \pm 0.10$ & 1 & $31.43 \pm 0.34$ & $-3.69 \pm 0.34$ & 16 \\
\hline HD 72688 & 5045 & $0.11 \pm 0.07$ & & $0.29 \pm 0.09$ & 2 & $-3.98 \pm 0.10$ & 2 & $30.96 \pm 0.07$ & $-4.30 \pm 0.07$ & 15 \\
\hline HD 83442 & 4715 & $0.02 \pm 0.10$ & & $1.06 \pm 0.13$ & 2 & $-3.64 \pm 0.10$ & 2 & $30.87 \pm 0.31$ & $-4.08 \pm 0.31$ & 14 \\
\hline HD 113816 & 4700 & $-0.11 \pm 0.09$ & $-0.28 \pm 0.13$ & $1.19 \pm 0.15$ & 2 & $-3.64 \pm 0.10$ & 2 & $31.09 \pm 0.28$ & $-4.16 \pm 0.28$ & 14 \\
\hline HD 118238 & 4575 & $-0.12 \pm 0.13$ & & $1.79 \pm 0.28$ & 2 & $-3.68 \pm 0.10$ & 2 & $31.66 \pm 0.51$ & $-4.27 \pm 0.51$ & 15 \\
\hline HD 119285 & 4770 & $-0.23 \pm 0.10$ & & $1.34 \pm 0.22$ & 2 & $-3.58 \pm 0.10$ & 2 & $30.56 \pm 0.09$ & $-3.77 \pm 0.09$ & 14 \\
\hline HD 181809 & 4902 & $-0.09 \pm 0.04$ & $-0.26 \pm 0.08$ & $0.55 \pm 0.08$ & 1 & $-3.82 \pm 0.10$ & 1 & $30.92 \pm 0.07$ & $-4.03 \pm 0.07$ & 14 \\
\hline HD 182776 & 4690 & $-0.06 \pm 0.16$ & & $1.26 \pm 0.36$ & 1 & $-3.78 \pm 0.11$ & 1 & $30.88 \pm 0.30$ & $-4.16 \pm 0.30$ & 17 \\
\hline HD 202134 & 4770 & $-0.06 \pm 0.07$ & $-0.19 \pm 0.11$ & $1.04 \pm 0.10$ & 1 & $-3.74 \pm 0.10$ & 1 & $30.99 \pm 0.15$ & $-3.91 \pm 0.15$ & 15 \\
\hline HD 204128 & 4845 & $-0.02 \pm 0.11$ & & $0.80 \pm 0.23$ & 1 & $-3.71 \pm 0.10$ & 1 & $31.50 \pm 0.37$ & $-3.25 \pm 0.37$ & 15 \\
\hline HD 205249 & 5055 & $0.12 \pm 0.09$ & & $0.59 \pm 0.17$ & 1 & $-3.69 \pm 0.10$ & 1 & $31.27 \pm 0.22$ & $-3.90 \pm 0.22$ & 16 \\
\hline HD 217188 & 4895 & $-0.16 \pm 0.07$ & & $0.72 \pm 0.12$ & 1 & $-3.79 \pm 0.10$ & 1 & $30.69 \pm 0.16$ & $-4.17 \pm 0.16$ & 16 \\
\hline \multicolumn{11}{|c|}{ Field subgiants } \\
\hline HD $1227^{\circ}$ & 5115 & $0.19 \pm 0.07$ & & $-0.07 \pm 0.11$ & 1 & & & $29.39 \pm 0.23$ & $-5.92 \pm 0.23$ & 18 \\
\hline HD 4482 & 4995 & $0.05 \pm 0.07$ & $-0.12 \pm 0.11$ & $0.27 \pm 0.11$ & 1 & $-4.23 \pm 0.10$ & 1 & $29.83 \pm 0.10$ & $-5.34 \pm 0.10$ & 18 \\
\hline HD 17006 & 5332 & $0.36 \pm 0.06$ & & $-0.16 \pm 0.07$ & 1 & $-4.31 \pm 0.10$ & 1 & $29.45 \pm 0.07$ & $-4.83 \pm 0.07$ & 18 \\
\hline HD 154619 & 5260 & $0.13 \pm 0.06$ & $-0.04 \pm 0.08$ & $-0.01 \pm 0.10$ & 1 & $-4.52 \pm 0.11$ & 1 & $29.52 \pm 0.20$ & $-5.72 \pm 0.20$ & 18 \\
\hline HD 156266 & 4665 & $0.25 \pm 0.08$ & $-0.28 \pm 0.11$ & & 1 & $-5.05 \pm 0.12$ & 1 & $28.97 \pm 0.18$ & $-6.46 \pm 0.18$ & 18 \\
\hline HD 211391 & 5025 & $0.23 \pm 0.07$ & $-0.18 \pm 0.11$ & $-0.08 \pm 0.08$ & 1 & & & $29.42 \pm 0.13$ & $-6.00 \pm 0.13$ & 18 \\
\hline HD 218527 & 5040 & $-0.08 \pm 0.09$ & & $0.10 \pm 0.22$ & 1 & & & $29.86 \pm 0.14$ & $-5.39 \pm 0.14$ & 18 \\
\hline \multicolumn{11}{|c|}{ Pleiades stars } \\
\hline HII 193 & 5339 & $0.06 \pm 0.05$ & & $0.22 \pm 0.08$ & 3 & & & $28.97 \pm 0.15$ & $-4.31 \pm 0.15$ & 19 \\
\hline HII 250 & 5715 & $0.06 \pm 0.05$ & & $0.11 \pm 0.08$ & 3 & & & $29.39 \pm 0.08$ & $-4.13 \pm 0.08$ & 20 \\
\hline HII 263 & 5048 & $0.06 \pm 0.05$ & & $0.89 \pm 0.08$ & 3 & & & $29.79 \pm 0.08$ & $-3.42 \pm 0.08$ & 19 \\
\hline HII 298 & 5048 & $0.06 \pm 0.05$ & & $0.50 \pm 0.08$ & 3 & & & $29.72 \pm 0.07$ & $-3.76 \pm 0.07$ & 19 \\
\hline HII 571 & 5373 & $0.06 \pm 0.05$ & $-0.04 \pm 0.11$ & $0.40 \pm 0.08$ & 3 & & & $<29.03$ & $<-4.30$ & 20 \\
\hline HII 676 & 4410 & $0.06 \pm 0.05$ & & 0.83 & 4 & & & $29.00 \pm 0.16$ & $-3.76 \pm 0.16$ & 20 \\
\hline HII 746 & 5407 & $0.06 \pm 0.05$ & & $0.28 \pm 0.08$ & 3 & & & $28.91 \pm 0.15$ & $-4.38 \pm 0.15$ & 19 \\
\hline HII 916 & 5098 & $0.06 \pm 0.05$ & & $0.59 \pm 0.08$ & 3 & & & $29.27 \pm 0.10$ & $-3.87 \pm 0.10$ & 19 \\
\hline HII 1593 & 5407 & $0.06 \pm 0.05$ & & $0.21 \pm 0.08$ & 3 & & & $<29.14$ & $<-4.21$ & 21 \\
\hline HII 2126 & 5142 & $0.06 \pm 0.05$ & & $0.31 \pm 0.08$ & 3 & & & $<29.33$ & $<-3.83$ & 21 \\
\hline HII 2284 & 5363 & $0.06 \pm 0.05$ & $-0.01 \pm 0.16$ & $0.31 \pm 0.08$ & 3 & & & $29.16 \pm 0.10$ & $-4.10 \pm 0.10$ & 19 \\
\hline HII 2311 & 5239 & $0.06 \pm 0.05$ & & $0.23 \pm 0.08$ & 3 & & & $28.82 \pm 0.15$ & $-4.45 \pm 0.15$ & 19 \\
\hline HII 2462 & 5174 & $0.06 \pm 0.05$ & & $0.53 \pm 0.08$ & 3 & & & $<28.53$ & $<-4.69$ & 19 \\
\hline HII 2880 & 5117 & $0.06 \pm 0.05$ & & $0.68 \pm 0.08$ & 3 & & & $28.98 \pm 0.11$ & $-4.17 \pm 0.11$ & 20 \\
\hline HII 3179 & 6172 & $0.06 \pm 0.05$ & & $0.01 \pm 0.08$ & 3 & & & $29.27 \pm 0.18$ & $-4.49 \pm 0.18$ & 20 \\
\hline \multicolumn{11}{|c|}{ Hyades stars } \\
\hline VB 15 & 5772 & $0.12 \pm 0.03$ & & $0.18 \pm 0.12$ & 5 & $-4.42 \pm 0.08$ & 10 & $29.11 \pm 0.09$ & $-4.48 \pm 0.09$ & 22 \\
\hline VB 19 & 6300 & $0.12 \pm 0.03$ & & $0.41 \pm 0.15$ & 6 & $-4.54 \pm 0.06$ & 10 & $29.29 \pm 0.09$ & $-4.69 \pm 0.09$ & 22 \\
\hline VB 25 & 4790 & $0.12 \pm 0.03$ & $0.04 \pm 0.08$ & & 5 & $-4.49 \pm 0.03$ & 10 & $<28.43$ & $<-4.67$ & 22 \\
\hline VB 31 & 6045 & $0.12 \pm 0.03$ & & $0.45 \pm 0.15$ & 6 & $-4.45 \pm 0.10$ & 10 & $29.09 \pm 0.09$ & $-4.76 \pm 0.09$ & 22 \\
\hline VB 48 & 6245 & $0.12 \pm 0.03$ & & $0.39 \pm 0.15$ & 6 & $-4.50 \pm 0.05$ & 10 & $29.28 \pm 0.07$ & $-4.64 \pm 0.07$ & 22 \\
\hline VB 65 & 6200 & $0.12 \pm 0.03$ & & $0.34 \pm 0.15$ & 6 & $-4.58 \pm 0.07$ & 10 & $29.06 \pm 0.08$ & $-4.80 \pm 0.08$ & 22 \\
\hline VB 66 & 6080 & $0.12 \pm 0.03$ & & $0.18 \pm 0.15$ & 6 & $-4.46 \pm 0.08$ & 10 & $29.30 \pm 0.07$ & $-4.56 \pm 0.07$ & 22 \\
\hline VB 73 & 5914 & $0.12 \pm 0.03$ & & $0.07 \pm 0.10$ & 5 & $-4.50 \pm 0.08$ & 10 & $29.06 \pm 0.09$ & $-4.63 \pm 0.09$ & 22 \\
\hline VB 79 & 5190 & $0.12 \pm 0.03$ & $0.02 \pm 0.08$ & & 5 & $-4.44 \pm 0.06$ & 10 & $28.63 \pm 0.16$ & $-4.71 \pm 0.16$ & 22 \\
\hline VB 88 & 6175 & $0.12 \pm 0.03$ & & $0.33 \pm 0.15$ & 6 & $-4.56 \pm 0.09$ & 10 & & & \\
\hline VB 97 & 5887 & $0.12 \pm 0.03$ & & $0.02 \pm 0.10$ & 5 & $-4.45 \pm 0.06$ & 10 & $29.00 \pm 0.13$ & $-4.76 \pm 0.13$ & 22 \\
\hline VB 105 & 6055 & $0.12 \pm 0.03$ & & $0.22 \pm 0.15$ & 6 & $-4.52 \pm 0.11$ & 10 & $29.15 \pm 0.08$ & $-4.52 \pm 0.08$ & 22 \\
\hline
\end{tabular}


T. Morel and G. Micela: Oxygen abundances in chromospherically active stars, Online Material p 3

Table 2. continued.

\begin{tabular}{|c|c|c|c|c|c|c|c|c|c|c|}
\hline $\mathrm{ID}^{a}$ & $\begin{array}{l}T_{\text {eff }} \\
(\mathrm{K}) \\
\end{array}$ & {$\left[\overline{\mathrm{Fe} / \mathrm{H}]^{b}}\right.$} & {$[\mathrm{O} / \mathrm{Fe}](6300)$} & [O/Fe] (Triplet) & Ref. & $\overline{\log \left(R_{\mathrm{HK}}^{\prime}\right)^{c}}$ & Ref. & $\begin{array}{c}\log \left(L_{X}\right)^{d} \\
\left(\mathrm{erg} \mathrm{s}^{-1}\right)\end{array}$ & $\overline{\log \left(R_{\mathrm{X}}\right)^{d}}$ & Ref. \\
\hline \multicolumn{11}{|c|}{ Field FG dwarfs } \\
\hline HD 3079 & 5892 & $-0.19 \pm 0.07$ & $-0.05 \pm 0.16$ & $0.39 \pm 0.16$ & 7 & -4.99 & 11 & & & \\
\hline HD 4614 & 5900 & $-0.27 \pm 0.10$ & $0.08 \pm 0.09$ & $0.08 \pm 0.11$ & 8 & -4.93 & 11 & $27.41 \pm 0.07$ & $-6.26 \pm 0.07$ & 23 \\
\hline HD 9562 & 5930 & $0.20 \pm 0.06$ & $-0.11 \pm 0.06$ & $-0.03 \pm 0.08$ & 9 & -5.19 & 11 & & & \\
\hline HD 10307 & 5883 & $0.02 \pm 0.10$ & $-0.02 \pm 0.07$ & $-0.22 \pm 0.12$ & 8 & -5.02 & 12 & $<27.64$ & $<-6.13$ & 24 \\
\hline HD 14412 & 5350 & $-0.47 \pm 0.06$ & $0.12 \pm 0.06$ & $0.15 \pm 0.08$ & 9 & -4.85 & 11 & $<27.08$ & $<-6.13$ & 25 \\
\hline HD 17051 & 6150 & $0.14 \pm 0.06$ & $-0.15 \pm 0.06$ & $0.11 \pm 0.08$ & 9 & -4.65 & 13 & $28.83 \pm 0.07$ & $-4.97 \pm 0.07$ & 23 \\
\hline HD 19994 & 6240 & $0.19 \pm 0.06$ & $-0.13 \pm 0.06$ & $0.04 \pm 0.08$ & 9 & -4.88 & 11 & & & \\
\hline HD 20630 & 5673 & $0.03 \pm 0.10$ & $0.01 \pm 0.15$ & $-0.11 \pm 0.11$ & 8 & -4.45 & 12 & $28.89 \pm 0.04$ & $-4.63 \pm 0.04$ & 23 \\
\hline HD 20807 & 5821 & $-0.21 \pm 0.10$ & $-0.10 \pm 0.12$ & -0.10 & 8 & -4.79 & 13 & $<27.14$ & $<-6.43$ & 25 \\
\hline HD 22484 & 5983 & $-0.10 \pm 0.10$ & $-0.02 \pm 0.08$ & 0.04 & 8 & -5.12 & 11 & $27.19 \pm 0.09$ & $-6.87 \pm 0.09$ & 24 \\
\hline HD 23249 & 5020 & $0.24 \pm 0.06$ & $-0.16 \pm 0.06$ & $-0.11 \pm 0.08$ & 9 & -5.22 & 13 & $26.95 \pm 0.18$ & $-7.14 \pm 0.18$ & 23 \\
\hline HD 26491 & 5745 & $-0.18 \pm 0.10$ & $-0.01 \pm 0.08$ & -0.06 & 8 & -4.95 & 13 & & & \\
\hline HD 30562 & 5926 & $0.14 \pm 0.10$ & $-0.10 \pm 0.08$ & -0.19 & 8 & -5.04 & 11 & & & \\
\hline HD 34411 & 5852 & $0.03 \pm 0.10$ & $0.01 \pm 0.09$ & $-0.12 \pm 0.10$ & 8 & -5.07 & 12 & $<27.40$ & $<-6.44$ & 24 \\
\hline HD 42618 & 5653 & $-0.16 \pm 0.07$ & $0.12 \pm 0.16$ & $0.30 \pm 0.16$ & 7 & -4.94 & 11 & & & \\
\hline HD 45184 & 5820 & $0.04 \pm 0.06$ & $-0.07 \pm 0.06$ & $0.11 \pm 0.08$ & 9 & -4.95 & 11 & & & \\
\hline HD 48938 & 6010 & $-0.37 \pm 0.10$ & $-0.01 \pm 0.09$ & -0.07 & 8 & -4.96 & 11 & & & \\
\hline HD 71148 & 5703 & $-0.08 \pm 0.07$ & $0.05 \pm 0.16$ & $0.27 \pm 0.16$ & 7 & -4.95 & 11 & & & \\
\hline HD 102870 & 6149 & $0.13 \pm 0.10$ & $-0.05 \pm 0.08$ & $-0.04 \pm 0.07$ & 8 & -4.94 & 11 & $28.39 \pm 0.04$ & $-5.74 \pm 0.04$ & 23 \\
\hline HD 108309 & 5710 & $0.10 \pm 0.10$ & $-0.03 \pm 0.09$ & -0.16 & 8 & -4.95 & 13 & & & \\
\hline HD 114710 & 6009 & $0.06 \pm 0.10$ & $-0.02 \pm 0.08$ & $-0.16 \pm 0.07$ & 8 & -4.76 & 11 & $28.06 \pm 0.06$ & $-5.66 \pm 0.06$ & 23 \\
\hline HD 121560 & 6081 & $-0.38 \pm 0.07$ & $0.17 \pm 0.16$ & $0.37 \pm 0.16$ & 7 & -4.92 & 11 & & & \\
\hline HD 141004 & 5915 & $-0.03 \pm 0.10$ & $0.16 \pm 0.05$ & $-0.05 \pm 0.08$ & 8 & -4.97 & 11 & $27.67 \pm 0.10$ & $-6.20 \pm 0.10$ & 23 \\
\hline HD 142860 & 6276 & $-0.14 \pm 0.10$ & $0.13 \pm 0.10$ & $-0.01 \pm 0.08$ & 8 & -4.82 & 11 & $27.28 \pm 0.15$ & $-6.76 \pm 0.15$ & 23 \\
\hline HD 144585 & 5880 & $0.33 \pm 0.06$ & $-0.21 \pm 0.06$ & $-0.05 \pm 0.08$ & 9 & -5.10 & 11 & & & \\
\hline HD 147513 & 5880 & $0.03 \pm 0.06$ & $-0.05 \pm 0.06$ & $-0.02 \pm 0.08$ & 9 & -4.52 & 13 & $28.96 \pm 0.03$ & $-4.61 \pm 0.03$ & 23 \\
\hline HD 147584 & 6090 & $-0.06 \pm 0.06$ & $-0.04 \pm 0.06$ & $0.12 \pm 0.08$ & 9 & -4.56 & 13 & $29.11 \pm 0.04$ & $-4.59 \pm 0.04$ & 23 \\
\hline HD 154417 & 6167 & $0.09 \pm 0.06$ & $-0.07 \pm 0.06$ & $0.00 \pm 0.08$ & 9 & -4.59 & 11 & $28.82 \pm 0.05$ & $-4.90 \pm 0.05$ & 23 \\
\hline HD 156365 & 5820 & $0.23 \pm 0.06$ & $-0.13 \pm 0.06$ & $0.10 \pm 0.08$ & 9 & -5.17 & 11 & & & \\
\hline HD 157347 & 5720 & $0.03 \pm 0.06$ & $-0.08 \pm 0.06$ & $-0.03 \pm 0.08$ & 9 & -5.04 & 11 & & & \\
\hline HD 157466 & 6050 & $-0.39 \pm 0.06$ & $0.08 \pm 0.06$ & $0.17 \pm 0.08$ & 9 & -4.89 & 11 & & & \\
\hline HD 160691 & 5800 & $0.32 \pm 0.06$ & $-0.23 \pm 0.06$ & $-0.03 \pm 0.08$ & 9 & -5.02 & 13 & $27.12 \pm 0.06$ & $-6.72 \pm 0.06$ & 25 \\
\hline HD 172051 & 5580 & $-0.24 \pm 0.06$ & $0.01 \pm 0.06$ & $0.06 \pm 0.08$ & 9 & -4.90 & 11 & $27.63 \pm 0.15$ & $-5.77 \pm 0.15$ & 23 \\
\hline HD 185144 & 5237 & $-0.24 \pm 0.10$ & $-0.07 \pm 0.16$ & $-0.04 \pm 0.13$ & 8 & -4.85 & 11 & $27.61 \pm 0.02$ & $-5.59 \pm 0.02$ & 23 \\
\hline HD 186408 & 5790 & $0.08 \pm 0.10$ & $-0.22 \pm 0.08$ & $-0.17 \pm 0.09$ & 8 & -5.10 & 11 & & & \\
\hline HD 186427 & 5719 & $0.03 \pm 0.10$ & $-0.09 \pm 0.08$ & $-0.17 \pm 0.10$ & 8 & -5.08 & 11 & & & \\
\hline HD 190248 & 5585 & $0.37 \pm 0.06$ & $-0.23 \pm 0.06$ & $0.00 \pm 0.08$ & 9 & -5.00 & 13 & $27.26 \pm 0.17$ & $-6.44 \pm 0.17$ & 23 \\
\hline HD 190406 & 5880 & $-0.11 \pm 0.10$ & $0.15 \pm 0.15$ & $0.05 \pm 0.08$ & 8 & -4.77 & 11 & $28.45 \pm 0.12$ & $-5.23 \pm 0.12$ & 23 \\
\hline HD 193307 & 5960 & $-0.32 \pm 0.06$ & $0.09 \pm 0.06$ & $0.34 \pm 0.08$ & 9 & -4.90 & 13 & & & \\
\hline HD 197214 & 5570 & $-0.22 \pm 0.06$ & $0.05 \pm 0.06$ & $0.12 \pm 0.08$ & 9 & -4.92 & 11 & $27.86 \pm 0.18$ & $-5.59 \pm 0.18$ & 26 \\
\hline HD 199960 & 5940 & $0.27 \pm 0.06$ & $-0.18 \pm 0.06$ & $-0.01 \pm 0.08$ & 9 & -5.08 & 11 & $27.76 \pm 0.18$ & $-6.12 \pm 0.18$ & 23 \\
\hline HD 206860 & 5926 & $0.03 \pm 0.10$ & $-0.04 \pm 0.10$ & $-0.10 \pm 0.09$ & 8 & -4.42 & 13 & $29.25 \pm 0.04$ & $-4.41 \pm 0.04$ & 23 \\
\hline HD 210277 & 5500 & $0.22 \pm 0.06$ & $-0.11 \pm 0.06$ & $0.07 \pm 0.08$ & 9 & -5.06 & 11 & & & \\
\hline HD 217014 & 5789 & $0.20 \pm 0.06$ & $-0.15 \pm 0.06$ & $-0.04 \pm 0.08$ & 9 & -5.08 & 11 & & & \\
\hline HD 217107 & 5620 & $0.35 \pm 0.06$ & $-0.24 \pm 0.06$ & $-0.03 \pm 0.08$ & 9 & -5.08 & 11 & & & \\
\hline HD 217877 & 5872 & $-0.18 \pm 0.07$ & $0.00 \pm 0.16$ & $0.34 \pm 0.16$ & 7 & -5.01 & 11 & & & \\
\hline HD 222368 & 6157 & $-0.21 \pm 0.10$ & $0.13 \pm 0.09$ & $0.21 \pm 0.05$ & 8 & -4.95 & 12 & $27.97 \pm 0.08$ & $-6.15 \pm 0.08$ & 23 \\
\hline HD 224022 & 6100 & $0.12 \pm 0.06$ & $-0.12 \pm 0.06$ & $0.09 \pm 0.08$ & 9 & -4.97 & 13 & & & \\
\hline
\end{tabular}

Key to references: [1] M04; [2] M03; [3] Schuler et al. (2004); [4] King et al. (2000); [5] King \& Hiltgen (1996); [6] García López et al. (1993); [7] Reddy et al. (2003); [8] King \& Boesgaard (1995); [9] Bensby et al. (2004); [10] Paulson et al. (2002); [11] Wright et al. (2004); [12] Soderblom (1985); [13] Henry et al. (1996); [14] Dempsey et al. (1993); [15] Dempsey et al. (1997); [16] Schwope et al. (2000); [17] Neuhäuser et al. (2000); [18] Hünsch et al. (1998a); [19] Micela et al. (1999); [20] Stauffer et al. (1994); [21] Micela et al. (1996); [22] Stern et al. (1995); [23] Hünsch et al. (1998b); [24] Schmitt \& Liefke (2003); [25] Schmitt (1997); [26] Hünsch et al. (1999).

${ }^{a}$ Identification for Pleiades and Hyades stars from Hertzsprung (1947) and van Bueren (1952), respectively.

${ }^{b}$ We quote the mean cluster metallicity of the Pleiades and Hyades determined by King et al. (2000) and Cayrel et al. (1985), respectively.

${ }^{c}$ For the RS CVn binaries and field subgiants, a blank indicates that the $\mathrm{Ca}$ II $\mathrm{H}+\mathrm{K}$ profiles were not filled in by emission. The errors take into account the uncertainties in $(V-R)_{0}$ and $T_{\text {eff }}$.

${ }^{d}$ Except for the open cluster stars and HD 118238 (130 pc; Strassmeier et al. 1993), all luminosities were rescaled to the Hipparcos distances. For consistency with previous X-ray observations, the bolometric luminosities were computed adopting a mean distance to the Pleiades of $127 \mathrm{pc}$ (Crawford \& Perry 1976). For the Hyades, we used the individual distances of Schwan (1991). No reddening and a mean extinction $A_{\mathrm{V}}=0.12 \mathrm{mag}$ were assumed for the Hyades and Pleiades, respectively (Nissen 1988). We used $A_{\mathrm{V}}=0.26$ mag for HII 676 (Stauffer et al. 1994). The bolometric corrections are taken from Flower (1996). The errors take into account the uncertainties in the X-ray counts and in the distances. 